paclitaxel or valspodar alone had no effect on tumour size.

Treatment of mice with valspodar alone for 46 days did not result in any observable systemic or CNS toxicity, indicating that this might be a useful approach for increasing the therapeutic efficacy of anticancer drugs that are P-GP substrates. Colon carcinoma and renal cancer cells have also been shown to express P-GP, so this combination therapy might also be used to increase the responsiveness of these tumours to chemotherapeutics.

Kristine Novak

(2) References and links ORIGINAL RESEARCH PAPER Fellner, S. et al. Transport of paclitaxel (Taxol) across the blood brain barrier in vitro and in vivo. J. Clin. Invest. 110 , 1309-1318 (2002)

FURTHER READING Rellin, M. V. \& Devieux, T. Pharmacogenetics and cancer therapy. Nature Rev. Cancer 1, 99-108 (2001) WEB SITE

Gert Fricker's lab: http://technologie.unihd.de/akfricker.htm

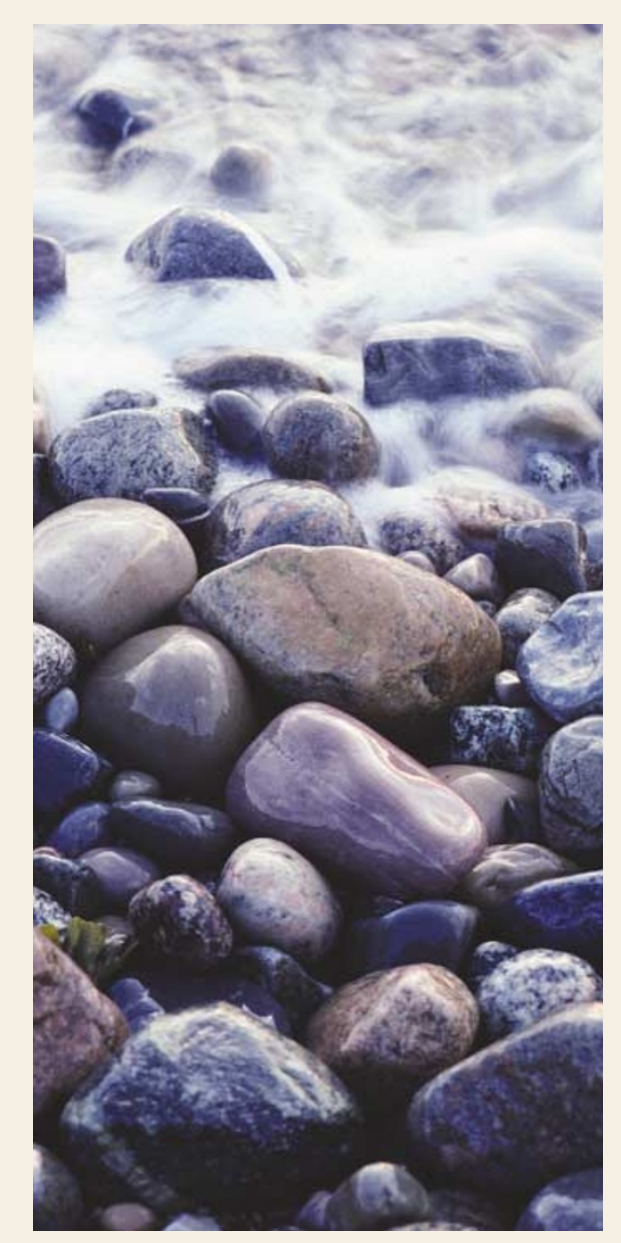

APOPTOSIS

\section{Escaping the rays}

Haematopoietic progenitor cells are often killed as a side effect of radiotherapy or chemotherapy and this is often the dose-limiting toxicity of cancer treatment. The understanding of how radiationinduced apoptosis is regulated in these cells has been poor, but now, in Cancer Cell, Thomas Look and colleagues provide some insight into how developing haematopoietic cells might escape radiation-induced death.

SLUG, a transcriptional repressor, is the human orthologue of the Caenorhabditis elegans celldeath specification protein CES1, and is a downstream target of E2A-HLF - a fusion oncoprotein that is found in a rare form of chemotherapy-resistant leukaemia. E2A-HLF is, itself, closely related to CES2, which acts upstream of CES1. So could SLUG have a regulatory role in the survival of haematopoietic progenitor cells?

Fluorescence-activated cell sorter (FACS) analysis showed that Slug is expressed by stem cells and early progenitor cells in mice, but not by the more mature haematopoietic cells, such as pro-B and pro-T cells. Look and colleagues exposed Slug-knockout mice to ionizing radiation, at a dose that killed $50 \%$ of the wildtype mice, and this caused premature death of all the knockout mice. Pathological examination of the mice showed that the mice had died of bonemarrow aplasia. They also observed that although Slug ${ }^{-/-}$mice had normal circulating blood cell counts, they had higher levels of haematopoietic colony-forming progenitor cells than wild-type mice, and they propose that cells with no Slug activate a feedback loop to maintain the normal numbers of the affected cells in the circulation.

Administration of a form of thrombopoietin - which was already known to enhance multilineage repopulation of the spleen after irradiation by preventing apoptosis in progenitor cells - protected the $\mathrm{Slug}^{-/-}$mice from death after irradiation. So, this indicated that progenitor cells in $\mathrm{Slug}^{-/}$mice normally undergo apoptosis and that Slug has a role in this cell-death mechanism.

To confirm this, the authors conducted a TUNEL assay in whole bone-marrow cells divided into two fractions - stem and early progenitor cells (about $12 \%$ of whole bone-marrow cells in both $\mathrm{Slug}^{-/}$and wild-type mice), and more mature haematopoietic cells. The mature cells from Slug ${ }^{-/}$ and wild-type mice were both resistant to apoptosis after ionizing radiation, whereas the progenitor cell fraction of $\mathrm{Slug}^{-/-}$mice had a much higher percentage of TUNEL-positive apoptotic cells compared with wild-type mice after irradiation. In addition, expression of Slug was

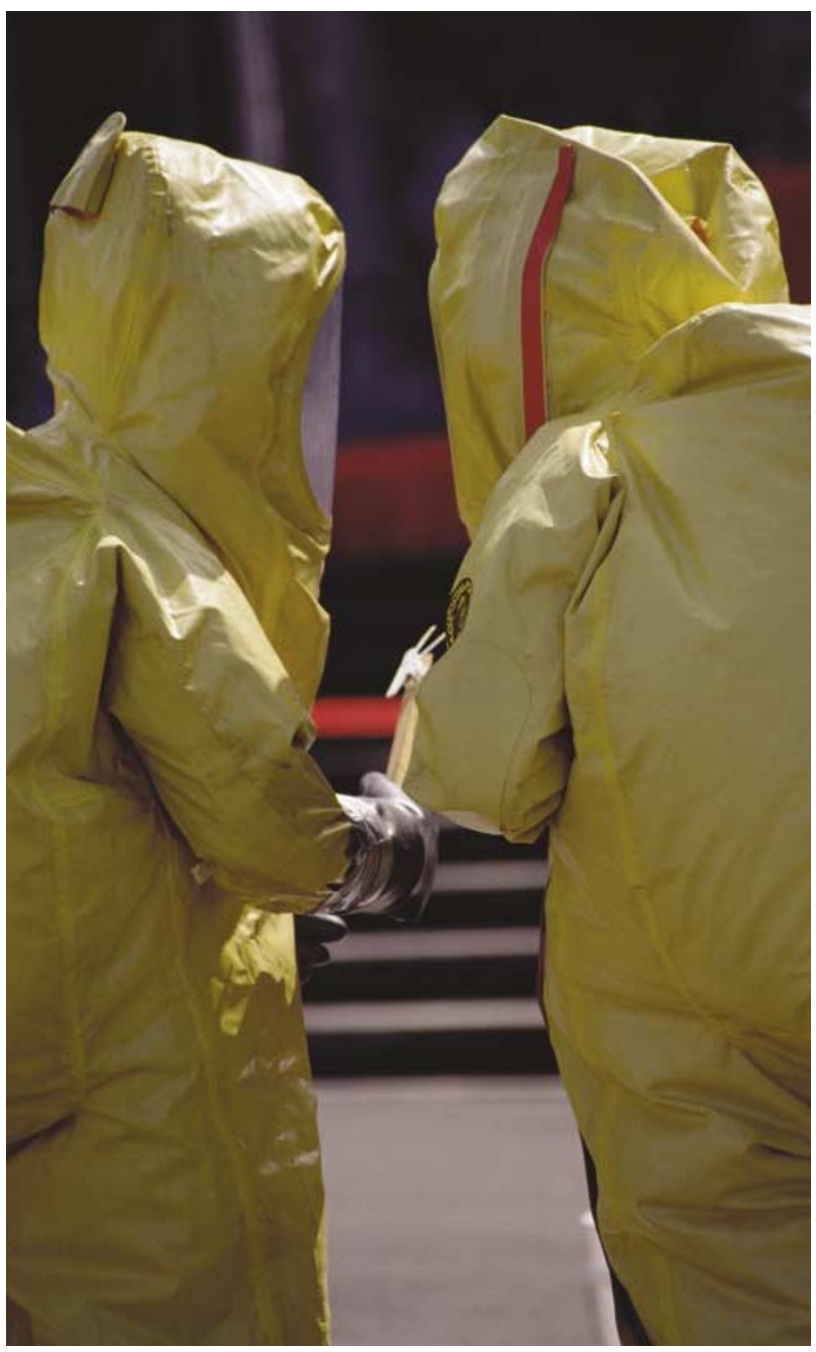

increased in the progenitor-cell population in wild-type mice following treatment, confirming that protection of progenitor cells from apoptosis is associated with upregulation of Slug.

The authors hypothesize that, due to similarities with the C. elegans cell-death pathway, Slug might function as a negative regulator of BH3-containing apoptotic proteins in differentiating haematopoietic cells. Defining the pathways through which Slug protects haematopoietic progenitor cells from apoptosis after DNA damage - which seems to act independently of p 53 - is the focus of the laboratory's current research. Exploitation of SLUG in cancer therapy to lower dose-limiting toxicity to haematopoietic cells of patients, and to possibly increase the therapeutic efficacy of cancer treatments, are future goals.

Ezzie Hutchinson

\section{(0) References and links}

ORIGINAL RESEARCH PAPER Inoue, A. et al. S/ug, a highly

conserved zinc finger transcriptional repressor, protects hematopoietic progenitor cells from radiation-induced apoptosis in vivo. Cancer Cell $\mathbf{2}$ 279-288 (2002)

WEB SITE

A. Thomas Look's lab: http://research.dfci.harvard.edu/looklab/ 